70 PAIN EXPERIENCED DURING VENIPUNCTURE IS HIGHER IN CHILDREN WITH A CHRONIC DISEASE

doi:10.1136/archdischild-2012-302724.0070

F Festini, C Dini, S Bisogni. University of Florence, Florence, Italy

Background Venipuncture pain in children results from several factors, which amplify the nociceptive stimulus; among them anticipatory anxiety plays an important role. Children with a chronic disease undergo invasive procedures and venipunture more frequently than other children.

Goal To evaluate whether a difference exists in pain threshold between children with a chronic disease and children with no previous experience of procedural pain.

Methods Cross-sectional analytical trial. A group of children with a chronic disease (group A) and a group of children with no previous experience of venipuncture (group B) aged 4 to 12 were observed during a standardized venipuncture for blood sample drawing. Pain was self-rated with a 1-10 Wong faces scale or a 1-10 numeric scale. Behavioural distress was measured with the Observational Behaviour Distress Scale (OBDS, 1-33).

Results 230 children were examined, 82 in group A and 148 in group $B$. With regards to pain, children in group A reported a mean pain score of 8.6 ( $\mathrm{sd} 1.2)$ while children in group B had a mean score of 3.1 (sd 2.4), Anova $p=0.00001$. With regards to behavioural distress, children in group A had a mean score of 26.7 (sd 4.3) vs. 8.3 in group B (sd 8.4), Anova $\mathrm{p}=0.00001$. The groups did not show statistically significant differences in age and sex distribution.

Discussion Children with a chronic disease, who previously underwent numerous venipunctures, experience a more intense pain and distress than their peers at their first experience of venipuncture.

\section{RELIABILITY AND VALIDITY OF TURKISH VERSION OF THE PHYSICAL ACTIVITY OUESTIONNAIRE FOR OLDER CHILDREN (PAQ-C)}

doi:10.1136/archdischild-2012-302724.0071

'L Erdim, ${ }^{2} \mathrm{~A}$ Ergun, ${ }^{3} \mathrm{~S}$ Kuguoglu. ${ }^{1}$ Pediatric Nursing Department, Istanbul University, Faculty of Health Science; ${ }^{2}$ Community Health Nursing, Marmara University, Faculty of Health Science, Division of Nursing, Istanbul, Turkey; ${ }^{3}$ Pediatric Nursing Department, Emeritus Professor from Marmara University, Faculty of Health Science, Division of Nursing, Brooklyn, NY, USA

Background and aims The aim of this study was to examine the psychometric properties of Turkish version of the Physical Activity Questionnaire for Older Children (PAQ-C) developed by Kowalski et al. The PAQ-C is a validated self-report measure of physical activity widely used to assess physical activity in children (8-14 years of age).

Methods This study was methodological design. The PAQ-C consists of nine questions was rated on a 5-point likert scale. The $\mathrm{PAQ}-\mathrm{C}$ was translated using a translation/back-translation method. The subjects were 549 Turkish school children. The internal consistency of the PAQ-C was assessed using Cronbach's alpha. Content validity was based on an experts' panel and assessed by using a content validity index

Results The study was completed with 549 children $(49.8 \%$ female, $50.2 \%$ male). Mean age of the participant was 10.30 years $(\mathrm{SD}=0.71$, range, $8-11$ years). The overall Content validity index (CVI) was $98.75 \%$ which signified that the PAQ-C has good content validity. Correlation values of scale items were found between $0.21-$ 0.70 for all items as the result of item total score analysis. Cronbach's alpha coefficient was found as 0.86 . The scale had a fairly good test-retest reliability $(r=0.64, \mathrm{p}<0.001)$.

Conclusions Psychometric analyses of the Turkish version of PAO-C indicate high reliability and good content and construct validity. The PAQ-C is a useful instrument for assess to physical activity patterns in Turkish children.

\section{INCIDENCE AND OUTCOME OF INFLICTED TRAUMATIC BRAIN INJURY AT THE PAEDIATRIC INTENSIVE CARE UNITS IN THE NETHERLANDS}

doi:10.1136/archdischild-2012-302724.0072

${ }^{1} \mathrm{~J}$ Elias, ${ }^{2} \mathrm{~N}$ Jansen, ${ }^{3 \mathrm{P}}$ van Hasselt. ${ }^{\mathrm{U}} \mathrm{UMC}$ Utrecht; ${ }^{2}$ Department of Pediatric Intensive Care; ${ }^{3}$ Department of Pediatric Metabolic Diseases, Wilhelmina Children's Hospital, Utrecht, The Netherlands

Background Inflicted traumatic brain injury (iTBI) is recognized as a major cause of death and poor outcome in young children.

Aim To determine the incidence and outcome of children with iTBI at the Paediatric Intensive Care Units (PICUs) in the Netherlands.

Methods The Dutch PICU registry was used to identify all children (0-2 years) who were admitted with traumatic brain injury (TBI) between 1-1-2005 and 31-12-2009. ITBI was defined as (1) proven with a confession or conviction, (2)confirmed by the Child protective services or (3)brain injuries, fractures or retinal hemorrhages incompatible with, or without history of trauma. Cases of iTBI were used to calculate the incidence for each year. Outcome parameters were compared for children with iTBI versus non-iTBI. Results During the study period 70 of 166 had iTBI (42\%), resulting in an overall incidence of 7.6/100.000 live births. The single year incidence varied markedly between 3.3 and 12.4 per 100.000 . The children with iTBI were younger than the children with non-iTBI (4.36 versus 7.49 months, $p<0.001$ ). Children with iTBI stayed significantly longer at the PICU (7.10 versus 3.92 days, $\mathrm{p}<0.01)$ and in hospital (27.62 versus 7.59 days, $p<0.001)$, and were significantly longer on mechanical ventilation (4.23 versus 1.65 days, $p<0.01$ ) iTBI was associated with a significantly higher mortality rate as compared with non-iTBI ( $23 \%$ vs $2 \%$, p<0.001).

Conclusions Of young children admitted to Dutch PICUs with TBI $42 \%$ had iTBI. The overall incidence of iTBI was 7.6/100.00. The children with iTBI had a significantly higher mortality rate of almost 25\%.

\section{HYPOTHERMIA-TREATED INFANTS WITH HYPOXIC- ISCHEMIC ENCEPHALOPATHY (HIE): MRI-FINDINGS CORRELATE WELL WITH NEUROMOTOR-OUTCOME AT 12 MONTHS}

doi:10.1136/archdischild-2012-302724.0073

${ }^{1} \mathrm{~K}$ Robertson Grossmann, ${ }^{2} \mathrm{~A}$ Tzovla, ${ }^{2} \mathrm{M}$ Kristoffersen Wiberg, ${ }^{1} \mathrm{~B}$ Hallberg. ${ }^{\prime}$ Neonatology; ${ }^{2}$ Radiology, Karolinska Institute, Karolinska University Hospital, Stockholm, Sweden

Background Induced hypothermia treatment (HT) for 72 hours reduces the risk of neurological sequele in asphyxiated full-term infants. Pathological findings on postnatal MRI of the brain have been shown to correlate to the prognosis.

This population-based study investigates the correlation between pathological findings in MRI of the brain after HT and neuromotor outcome at 12 months of age in asphyxiated full-term infants with HIE.

Methods In Stockholm, between January 2006 and December 2009, all infants fulfilling A- and B-criteria for HT were included. MRI of the brain was done within 2 weeks from birth. Neurological assessment was done at 4 and 12 months of age.

Results Of the 70 infants who received HT, both MRI examination and outcome data were available for 60 infants. Patterns of injury on MRI were defined based on the predominant site of injury: Watershed predominant (WS), basal ganglia/thalamus predominant $(\mathrm{BG} / \mathrm{T})$, general/global lesions $(\mathrm{G})$ and normal. 
46 infants had normal neuromotor outcome at 12 months of age. 14 infants had abnormal outcome ( 2 mild/moderate impairment, 12 cerebral palsy, 4 ad mortem).

\section{Abstract 73 Table 1}

\begin{tabular}{lcccc}
\hline MRI-findings & Normal 61,7\% & WS $\mathbf{1 6 , 7 \%}$ & BG/T 18,4\% & G 3,3\% \\
\hline Normal outcome & 37 & 8 & 1 & - \\
Mild/moderate impairment & - & 1 & 1 & - \\
Severe impairment/death & - & 1 & 9 & 2 \\
\hline
\end{tabular}

Conclusions The development and severity of motor deficits due to perinatal asphyxia correlates to the pattern of brain injury seen on MRI. MRI provides valuable prognostic information in hypothermia-treated infants.

\section{TRAINING AND CHECKLISTS; HOW TO SAFELY EVACUATE A NICU}

doi:10.1136/archdischild-2012-302724.0074

J van Duuren, C Lakke, B Peels, A van den Hoogen, D Vijlbrief. Neonatology, UMC Utrecht, Utrecht, The Netherlands

Introduction Evacuation is an uncommon and hard to practice event in a Neonatal Intensive Care Unit (NICU). Insecurity of untrained personnel can lead to disastrous incidents. To improve training and to recognize problems, a large multidisciplinary evacuation training was organized.

Methods The evacuation procedure was filmed by a professional film crew and edited into a 10-minute instructional movie. The film was shown to the full nursing staff. With lessons learned from this evacuation the current evacuation protocol was evaluated and several inconsistencies were identified.

Results During the training the current evacuation area proved unsuitable because of absence of the appropriate connections. Furthermore the staff trained was uncertain of their tasks during the training. Lastly essential equipment was hard to find. To increase awareness and regulate the procedure a checklist was developed. The coordinating nurse was made responsible for a weekly run through of the checklist. The instructional video will be shown to all new colleagues. Currently, a simulation computer program is being developed to routinely practice an evacuation in a safe environment.

Conclusion The local evacuation procedure should be well known among NICU staff. Multidisciplinary training is an important tool to identify the positive and negative aspects of the current procedure. A checklist can help to increase awareness and to the early identification of possible problems.

Acknowledgements Multidisciplinary working group evacuation, the fire department UMC Utrecht and fire department Utrecht.

\section{AGE APPROPRIATE CARE OF THE PREMATURE AND HOSPITALIZED INFANT: NURSING WORKFLOW EFFICIENCIES AND OUALITY CLINICAL OUTCOMES}

doi:10.1136/archdischild-2012-302724.0075

M Coughlin. Interprofessional Education, Caring Essentials Collaborative, Inc., Boston, MA, USA

Background and aim Developmental care has been shown to decrease the length of hospital stay and hospital costs, improve weight gain and time to full enteral feeds as well as improve neurodevelopmental scores at 9-12 months. Despite these benefits, there is inconsistent definition, application, and evaluation of developmental care practices in the neonatal ICU. The core measures for developmentally supportive care were developed as an evidence based standard to mitigate the existing inconsistencies and have now been adopted by National Association of Neonatal Nurses (USA) as their new Guidelines for Age Appropriate Care of the Premature and Hospitalized Infant. This abstract will present the impact of these guidelines for practice on nursing workflow efficiencies as well as infant clinical outcomes.

Methods Using an observational cohort, a transformational educational intervention was introduced to a level III NICU framed by the core measures for developmental care. Clinical outcomes for infants less than 32 weeks gestation, NICU bed occupancy, NICU nursing FTEs and NICU patient length of stay are the measured variables.

Results Despite an increase in bed occupancy during the study period there was a decrease in patient length of stay with essentially the same number of nursing FTEs in both the benchmark group and the study group. Key clinical morbidities were significantly reduced as well.

Conclusion Clearly defined, measurable objectives as defined by the core measures for developmentally supportive care favorably impacts not only clinical outcomes but also nursing workflow efficiencies and nursing satisfaction.

\section{VIRAL INFECTIONS IN NEONATES}

doi:10.1136/archdischild-2012-302724.0076

MA Verboon-Maciolek. Neonatology, University Medical Center Utrecht, Utrecht, The Netherlands

Infections are an important cause of mortality and morbidity in the neonatal period. In the past viral infections were difficult to diagnose. Since the introduction of sensitive molecular methods such as polymerase chain reaction (PCR) in the identification of viruses the laboratory diagnosis of viral infections in neonates has been improved.

Viruses can be transmitted vertically (during the pregnancy or delivery) or horizontally (after birth). Cytomegalovirus is the most common cause of intrauterine infection worldwide leading to neurodevelopmental sequelae and hearing deficits. Herpes simplex virus (HSV) is the important cause of perinatal (during the delivery) or postnatal (after birth) infection. Recently it has been shown that various viruses which are transmitted horizontally (after birth) may also lead to life-threatening diseases such as sepsis and encephalitis. The recognition of these infections may be difficult because of their similarity with bacterial infections. The diagnosis can only be made if the proper PCR is performed. The consequences of systemic enterovirus, parechovirus or rotavirus infection in the neonatal period may be disastrous. In many infants severe white matter damage was documented on cerebral MRI, subsequently leading to neurodevelopmental delay. Because antiviral treatment is not available, preventive measures such as adequate hand hygiene must be taken into account in care of newborn infants.

During the presentation the epidemiology, clinical presentation, neuro-imaging (cerebral ultrasonography and MRI) and outcome of infants with various viral infections will be presented. Furthermore the preventive measures will be discussed.

\section{COMPARISION OF TWO DIFFERENT DISTRACTION METHODS ON PAIN RELIEF OF CHILDREN DURING BLOOD DRAW}

doi:10.1136/archdischild-2012-302724.0077

'N Canbulat, ${ }^{2} \mathrm{~S}$ I nal, 'H Sönmezer. 'Karamanoglu Mehmetbey University School of Health, Karaman; ${ }^{2}$ stanbul University Health Science Faculty, Istanbul, Turkey

Background and aims The procedures made by a needle are the most prevalent and important sources of pain for children. Then, this study aims to compare the effects of distraction by way of 